\title{
A NOTE ON ASYMPTOTIC INFERENCE FOR AN ASYMMETRIC ISING MODEL AROUND A TORUS
}

\author{
WEI QIAN, ${ }^{*}$ University of Glasgow
}

\begin{abstract}
Limiting distribution results are obtained for the sufficient statistics of an asymmetric Ising model on a torus. Applications are discussed.
\end{abstract}

\section{Introduction}

Consider a rectangular lattice with $M \times N$ pixels. Let $X=\left(x_{i j}\right)$ denote the vector variable with each $x_{i j} \in\{-1,+1\}$. We assume a periodic boundary condition which is equivalent to wrapping the lattice around a torus. The distribution function for the periodic boundary asymmetric Ising model, which involves two parameters, is

$$
P(X \mid \alpha, \beta)=\frac{1}{C(\alpha, \beta)} \exp \left\{\alpha \sum_{i=1}^{M} \sum_{j=1}^{N} x_{i j} x_{i, j+1}+\beta \sum_{i=1}^{M} \sum_{j=1}^{N} x_{i j} x_{i+1, j}\right\}
$$

where $x_{i, N+1}=x_{i 1}$ and $x_{M+1, j}=x_{1 j}$, and $C(\alpha, \beta)$ is the normalizing factor known as the partition function. Define $Q=\left(Q_{1}, Q_{2}\right)^{\prime}=\left(\sum \sum x_{i j} x_{i, j+1}, \sum \sum x_{i j} x_{i+1, j}\right)^{\prime}$. For convenience, we only consider the region $R_{+}^{2}=\{0 \leqq \alpha, \beta<\infty\}$. The results can also be extended to other areas by symmetric extension.

\section{The limiting results of $Q$}

The moment generating function of $Q$ is $C\left(\alpha+t_{1}, \beta+t_{2}\right) / C(\alpha, \beta)$. The asymptotic properties of $Q$ depend upon those of $C(\alpha, \beta)$. There is a function, $B(\alpha, \beta)$, which is an approximation to $(M N)^{-1} \log C(\alpha, \beta)$ and defined by the following Riemann integral. Detailed proof of the validity of the approximation is not given here, but the approach is similar to that of Pickard (1976):

where

$$
B(\alpha, \beta)=(4 \pi)^{-1} \int_{0}^{2 \pi} \psi(\alpha, \beta, \omega) d \omega,
$$

$$
\begin{aligned}
\psi(\alpha, \beta \omega)= & \log \{\cosh 2 \alpha \cosh 2 \beta-\sinh 2 \alpha \cos \omega \\
& \left.+\left[(\cosh 2 \alpha \cosh 2 \beta-\sinh 2 \alpha \cos \omega)^{2}-(\sinh 2 \beta)^{2}\right]^{\frac{1}{2}}\right\} .
\end{aligned}
$$

It is not difficult to show that there is a line, called the critical line, and defined by

$$
L=\left\{(\alpha, \beta)^{\prime}: \sinh 2 \alpha \sinh 2 \beta=1\right\}
$$

such that, although the first-order partial derivatives of $B$ are continuous on $R_{+}^{2}$, the high-order partial derivatives are not defined on $L$. By using Kaufmann's (1949) exact representation for $C(\alpha, \beta)$, Pickard (1976) obtained the limiting distribution properties of $Q_{1}+Q_{2}$, but only for the symmetric case $\alpha=\beta$. Following almost the same method and notations as those of Pickard (1976) to analyse $C(\alpha, \beta)$, and then using the moment generating functions of $(M N)^{-1} Q$ and $(M N)^{\frac{1}{2}}\left[(M N)^{-1} Q-\nabla B(\alpha, \beta)\right]$, we can prove the following theorem.

Received 31 August 1989; revision received 15 May 1990.

* Postal address: Department of Statistics, University of Glasgow, Glasgow G12 8QQ, Scotland, UK. 
Theorem. Suppose $(\alpha, \beta)^{\prime} \notin L$. Then as $M, N \rightarrow \infty$, and provided $N^{\theta 1} \leqq M \leqq N^{\theta}$ for any fixed $\theta_{1}$ and $\theta$ with $0<\theta_{1}<\theta<\infty$,

1. $(M N)^{-1} Q \stackrel{\operatorname{Pr}}{\longrightarrow} \nabla B(\alpha, \beta)$

2. $(M N)^{\frac{1}{2}}\left[(M N)^{-1} Q-\nabla B(\alpha, \beta)\right] \stackrel{\mathrm{D}}{\longrightarrow} \mathrm{N}\left(0, \nabla^{2} B(\alpha, \beta)\right)$

where $\nabla$ denotes the first-order derivative vector, and $\nabla^{2}$ the second-order derivative matrix.

For the case of free boundary condition (no interaction between $x_{i 1}$ and $x_{i N}$, and between $x_{1 j}$ and $x_{M j}$ ), it is almost impossible to analyse the partition function exactly in order to obtain rate of convergence of $(M N)^{-1} E Q$ to $\nabla B(\alpha, \beta)$. Pickard (1977) showed only that

$$
(M N)^{-\frac{1}{2}}[Q-E Q] \stackrel{\mathrm{D}}{\longrightarrow} \mathrm{N}\left(0, \nabla^{2} B(\alpha, \beta)\right) .
$$

\section{Applications}

Since the number of possible terms of $X$ is $2^{M N}$, it is not feasible to carry out an exact calculation for $C(\alpha, \beta)$ and $\nabla C(\alpha, \beta)$, except when $M$ and $N$ are small. The maximization of the log-likelihood is therefore infeasible. Monte Carlo methods are not a completely satisfactory approach, since the author believes the computational burden of simulating the field to be very heavy. Note that $M N B(\alpha, \beta)$ is an approximation to $\log C(\alpha, \beta)$, an obvious alternative way of estimating parameters is to maximize an asymptotic likelihood, namely, $A L(Q \mid \alpha, \beta)=\alpha Q_{1}+\beta Q_{2}-M N B(\alpha, \beta)$, or to solve the following corresponding asymptotic normal equation:

$$
(M N)^{-1} Q=\nabla B(\alpha, \beta) \text {. }
$$

Suppose $\left(\alpha_{0}, \beta_{0}\right)^{\prime} \notin L$ are the true values of the parameters and denote by $(\hat{\alpha}, \hat{\beta})^{\prime}$ the solution of the above equation. Standard methods therefore show that as $M, N \rightarrow \infty$, and provided $N^{\theta_{1}} \leqq M \leqq N^{\theta}$ with $0<\theta_{1}<\theta<\infty$,

$$
(M N)^{-1}\left(\begin{array}{c}
\hat{\alpha}-\alpha_{0} \\
\hat{\beta}-\beta_{0}
\end{array}\right) \stackrel{\mathrm{D}}{\longrightarrow} \mathrm{N}\left(0,\left(\nabla^{2} B(\alpha, \beta)\right)^{-1}\right) .
$$

This demonstrates consistency of $(\hat{\alpha}, \hat{\beta})^{\prime}$. Another application of the results is in testing the null hypothesis:

$$
\mathrm{H}_{0}: \alpha=\beta
$$

against the general alternative hypothesis of 'not $\mathbf{H}_{0}$ '. We can naturally attack this test problem by an asymptotic likelihood-ratio approach. Define the test statistic

$$
\Lambda(Q)=\sup _{\alpha, \beta} A L(Q \mid \alpha, \beta)-\sup _{\alpha} A L(Q \mid \alpha, \alpha) .
$$

The upper $\delta$-point $q_{\delta}$ of the distribution can then be defined by

$$
\max _{(\alpha, \alpha) \notin L} \lim _{M, N \rightarrow \infty} \operatorname{Pr}\left[\Lambda(Q) \geqq q_{\delta} \mid \alpha, \alpha\right]=\delta .
$$

It can be shown that, when the null hypothesis holds, $\Lambda(Q)$ follows, asymptotically, a $\chi^{2}(1)$ distribution, while, when the alternative hypothesis holds, $\Lambda(Q)$ has. in probability, the same order as $M N$. For the free boundary condition case, the asymptotic normality of maximum asymptotic likelihood estimators and the properties of above test statistic have not been established. 


\section{Acknowledgement}

The author is grateful to Professor D. M. Titterington for his advice and support. He also thanks the University of Glasgow and the ORS Committee for financial support.

\section{References}

Kaufmann, B. (1949) Crystal statistics II, partition function evaluated by spinar analysis. Phys. Rev. 76, 1232-1243.

Pickard, D. K. (1976) Asymptotic inference for an Ising lattice. J. Appl. Prob. 13, 486-497.

PicKard, D. K. (1977) Asymptotic inference for an Ising lattice II. Adv. Appl. Prob. 9, 476-501. 